\title{
Un entrenamiento para las tareas: perspectiva desarrollista en la Educación Física Argentina del Nivel Inicial
}

A Task Training: developmental perspective in the Argentine Physical Education of the Initial Level

\author{
Daniela Mansi \\ Orcid: https://orcid.org/0000-0001-8060-811X \\ Universidad Nacional de Luján - Universidad de Flores, Luján, Argentina, \\ Danielamansi19@gmail.com
}

Received on 27/04/2020 - Approved on 07/05/2020

\section{Resumen}

El corriente escrito porta como objetivo general, desarrollar y caracterizar la perspectiva desarrollista de la Educación Física Argentina en el Nivel Inicial. Se ha llevado adelante un estudio enmarcado en la metodología cualitativa, siendo una pesquisa exploratoria - descriptiva, posicionada desde un enfoque interpretativo. Los documentos tomados como muestra, la cual ha sido intencional - finalística, han sido competentes a la didáctica de la Educación Física en el Nivel Inicial en Argentina. En base al análisis demostramos que la perspectiva desarrollista, encontrada a partir del año 1966, opera desde la pedagogía tecnicista, legitimando propuestas corporales infantiles sobre los mandatos del rendimiento, la eficacia y la eficiencia.

Palabras claves: Perspectiva Desarrollista. Educación Física. Nivel Inicial.

\section{Abstract}

The general objective of the written trend is to develop and characterize the developmental perspective of Argentine Physical Education at the Early Level. A study has been carried out within the framework of qualitative methodology, being an exploratory-descriptive research, positioned from an interpretative approach. The documents taken as a sample, which have been intentional - finalistic, have been competent to the didactics of Physical Education in the Initial Level in Argentina. Based on the analysis, we show that the developmentalist perspective, found since 1966, operates from the technical pedagogy, legitimizing children's body proposals on the mandates of performance, efficacy and efficiency.

Key words: Developmentalism perspective. Physical Education. Initial Level. 


\section{Introducción}

A partir de la Ley de Educación Común $N^{\circ} 1420$ del año 1884, y consecuentemente la sistematización del sistema educativo argentino, comienza a impartirse en las escuelas argentinas la materia Educación Física, fuertemente militarizada, y legitimada por la corriente positivista decimonónica una vez afianzada como espacio curricular. En el correr de su cronología, los gobiernos epocales han encontrado en la asignatura el espacio propicio para la construcción de los arquetipos de cuerpos infantiles deseados para la nación.

No obstante, tomando los aportes de Bracht (1996) diremos que la Educación Física se ha ido construyendo como materia escolar obligatoria en función de visiones heterónomas y autónomas. Éstas primeras, remiten a la necesidad que tuvo (y tiene) la Educación Física en tomar los aportes de instituciones ajenas al campo mismo de la asignatura (institución militar, deportiva y médica), y las visiones autónomas como aquellas conformadas desde el núcleo de la Educación Física, realizando así independencia del campo.

Estudios posteriores, han demostrado que la Educación Física Argentina se ha anclado de distintos ámbitos de referencia para legitimar su posicionamiento en las instituciones escolares (Aisenstein, 2006; Aisenstein y Scharagrodsky, 2006; Beer, 2013; Galak y Gambarotta, 2015; Orbuch, 2016; Rozengardt y Acosta, 2006; Scharagrodsky, 2004, 2008, 2011, 2014, 2015), o bien para responder ante las políticas educativas de los gobiernos de turno.

Las pesquisas nombradas, remiten a la historicidad de la Educación Física específicamente en el nivel primario y medio, siendo Villa (2006) y Mansi (2018) quienes enfocaron sus estudios en el nivel inicial. Haciendo bases sobre sus resultados, hemos detectado que se presentan en el transcurso cronológico (19432015) de la Educación Física del Nivel Inicial las siguientes perspectivas ideológicas: higienista, psicomotricista, desarrollista, recreacionista, deportivista en menor medida, y la visión humanista con rasgos socio-críticos.

En el presente escrito, nos remitiremos a la perspectiva desarrollista la cual hallamos arraigada a una pedagogía tecnicista puesta en práctica sobre los cuerpos infantiles, mediante la búsqueda del rendimiento, eficiencia y eficacia motriz.

De tal manera, el corriente artículo, portará como objetivo general desarrollar y caracterizar la perspectiva desarrollista ${ }^{1}$ de la Educación Física del Nivel Inicial en Argentina, la cual se encuentra en aparición dentro de los documentos a partir del año 1966 y su aumento cuantitativo en la década del '70, específicamente luego del autollamado Proceso de Reorganización Nacional y la puesta en práctica de políticas educativas autoritarias.

\section{Diseño metodológico}

La investigación ha sido abordada desde un enfoque cualitativo (Denzin y Lincoln, 2011) dicho término implica un énfasis en las cualidades de los entes a investigar, en los procesos y sus significados. Diremos entonces, que hemos 
colocado nuestro foco en aquellas características que ha de tener nuestro objeto de estudio, siendo el diseño de tipo exploratorio - descriptivo (Samaja, 1999).

La pesquisa se ha hallado enmarcada en un esquema interpretativo, en el cual el objeto a estudiar se lo aborda como un mensaje a descifrar (Ynoub, 2015). Continuando con la autora, estas investigaciones se realizan en base al contexto histórico del objeto.

El muestreo ha de ser intencional y finalístico (Ynoub, 2015). Hemos tomado como muestra, 17 libros, 13 artículos de revista Editorial Stadium, y 1 investigación doctoral, competentes a la didáctica de la Educación Física en el Nivel Inicial en Argentina. De ellos, hemos analizado los objetivos, los contenidos, las expectativas de logro y la función social que cada autora y autor le otorgan a la Educación Física del Nivel Inicial en su bibliografía.

Las fuentes seleccionadas comparten afinidad y pertinencia con el objeto de estudio, adhiriéndonos a la idea que los libros, revistas, e investigaciones se transforman en un excelente corpus de ser analizados y en un fuerte vector de vehiculización ideológica.

Desde allí, tomamos a la documentación seleccionada como una estrategia metodológica de información (Valles, 1999).

\section{Resultados y discusiones}

\section{Caracterizaciones de la perspectiva desarrollista de la Educación Física en el nivel inicial: del contexto socio político a sus propuestas}

Como nombramos en líneas anteriores, nuestra investigación se encuentra sostenida en el enfoque interpretativo (Ynoub, 2015), desde el cual, para lograr un mejor estudio de nuestro objeto, nos hallamos en la necesidad de esclarecer el marco político - social epocal.

Cabe destacar, en base a los resultados de la investigación, que la perspectiva desarrollista de la Educación Física en el nivel inicial, encuentra su auge, legitimación y conformación en base a dos hechos: en cuanto a las prácticas corporales en la década de 1970, inmigra a Argentina la rama psicomotricista educativa, interviniendo en las propuestas pedagógicas de los y las docentes del área, y aportándole a la misma una fuerte adherencia científica. Ello genera una dependencia de la materia hacia la psicomotricidad.

Y otro, en la misma década, asume en Argentina un gobierno de facto, dando inicio a años de autoritarismo, persecución, secuestros y desapariciones. Y dentro del ámbito educativo una fuerte censura y puesta en práctica el tecnicismo pedagógico.

No obstante, desarrollaremos brevemente en líneas subsiguientes ambos hechos que dan pie a la explosión cuantitativa bibliográfica de la Educación Física en el nivel inicial correspondiente a la perspectiva desarrollista de la misma.

Como hemos sostenido, la vertiente desarrollista de la Educación Física comienza a legitimarse en el correr de la década de 1970, momento en el cual recorre en Argentina una de las dictaduras más atroces que la sociedad ha vivido. 
El 24 de marzo de 1976 interrumpen el mando del gobierno, la Junta de Comandantes en Jefe, encabezada por Videla, Massera y Agosti, quienes dieron inicio al autollamado Proceso de Reorganización Nacional.

Tomando a Puiggrós (1992) la trama político - cultural argentina estaba completamente arraigada a la creencia que el orden autoritario solucionaría los problemas sociales. Dicho orden, se impregna en las instituciones educativas, tanto en el nivel inicial, primario y medio, como terciario y universitario.

En consecuencia, con la ideología arraigada en el autoritarismo, la educación formal en general, y el nivel inicial en particular han sido los espacios institucionalizados que se encontraron bajo la desconfianza de ser vehiculizadores de ideología comunista y subversiva.

Fernández Pais (2019) nos interpela diciendo que a partir de 1976 el jardín de infantes se encontraba bajo sospecha, lugar donde podría germinar la subversión, y florecer el futuro caos social. Es por ello, que en el año 1977 se difunde un documento generado y distribuido a los establecimientos educacionales por el Ministerio de Cultura y Educación ${ }^{2}$ con intenciones de ser leídos por docentes y estudiantes (si fuese necesario), quienes debían estar atentos/as a posibles ideologías subversivas.

Por consiguiente, con un nivel inicial bajo sospecha, y con una pedagogía tecnicista puesta en práctica, la Educación Física del primer eslabón educativo, se haya en concordancia a las políticas educativas de turno, específicamente en vistas de, mediante sus propuestas pedagógicas, la búsqueda de los valores que el tecnicismo pedagógico trae consigo: la eficiencia, la eficacia y el rendimiento infantil.

No obstante, no sólo las políticas educativas de turno han generado que la perspectiva desarrollista se legitime, sino que la misma hace sus bases sobre la educación psicomotora. Por ende, se nos genera la mera necesidad de manifestar la llegada de la educación psicomotriz a nuestro país en 1970, y sus injerencias dentro de la pedagogía de la Educación Física específicamente en el nivel inicial.

Sin ampliar sobre la temática, comentaremos que la perspectiva psicomotricista propone una educación integral de los/as sujetos, pensando en educar lo cognitivo, lo motriz, lo emocional y lo afectivo, de manera conjunta. Hasta impensado en momentos anteriores, la vertiente psicomotora, específicamente la rama de la psicokinética creada por Le Boulch (1978), presenta una educación integral, creando el método psicocinético el cual "utiliza el movimiento humano para educar" (Le Boulch, 1978, p. 11).

Resumiendo y esclareciendo los acontecimientos contextuales, en el correr de la década de 1970, sucede a nivel socio-político un golpe cívico-militar en Argentina, generando políticas sociales y educativas meramente autoritarias. $Y$ en el campo de la Educación Física, se recibe a la psicomotricidad como ciencia que contribuye a las argumentaciones teóricas de la asignatura.

Una vez comentado los dos hechos significativos para nuestro escrito, que han penetrado y hecho cenizas en las instituciones educativas, pasaremos en el correr de las líneas a vislumbrar las características de la perspectiva desarrollista de Educación Física Argentina en el Nivel Inicial.

Creemos propicio destacar, que la categoría ha sido aportada por Falkenbach, Drexsler y Werle (2006) quienes han realizado una investigación acerca de cómo se estructuran pedagógicamente las clases de Educación Física 
infantil en Brasil. Uno de los aportes enriquecedores, es que a partir de la pesquisa han detectado que la perspectiva desarrollista, encargada de la enseñanza de las habilidades motrices, se encuentra atravesada en las prácticas pedagógicas de la Educación Física en el nivel inicial en Brasil.

Tomando dicha categoría, extrapolaremos los datos hacia la Educación Física Argentina en la educación inicial, analizando la bibliografía competente a la didáctica de la Educación Física en el nivel inicial.

Desde la Guía de Educación Física para los jardines de infantes, Marrazzo y Marrazzo (1970), proponen como contenidos: Formas básicas primarias, formas básicas secundarias, movimientos con elementos, movimientos con aparatos.

En base a ello, diremos que el eje de las clases de Educación Física en el nivel inicial se encuentra en preponderar el movimiento en la niñez. Continuando, Giraldes y Porstein (1976) nos proponen que es "en la etapa pre-escolar un objetivo predominante: la formación motriz" (Giraldes y Porstein, 1976, p. 46).

Haciendo bases sobre los aportes de González y Gómez (1978), uno de los libros más vendidos desde la Editorial Stadium (arrojándonos que hasta el año 2010 hubo 30.000 ejemplares vendidos), los contenidos de la Educación Física en la infancia deben ser:

- Las acciones motrices con acento colocado en la fuerza, velocidad, resistencia y flexibilidad.

- El espacio-tiempo: orientación.

- El propio cuerpo.

- Los desplazamientos, saltos, apoyos, rolidos, trepas, suspensiones, equilibrio, empuje, tracción, lanzamiento, pase, recepción.

- Juegos libres y reglados.

En función de las manifestaciones de los/as autores/as, se deja entrevisto que la funcionalidad ofrecida a la Educación Física en el nivel inicial se encuentra bajo la enseñanza de las habilidades motoras básicas, colocando a la asignatura como dadora de tareas motrices. Se vislumbra una preponderancia hacia el movimiento infantil, desterrando todo tipo de quietud e inacción durante el espacio curricular de Educación Física.

No obstante, hemos de hallar entre los contenidos ofrecidos, una fuerte adherencia a la perspectiva psicomotora, tomando el desarrollo del equilibrio, esquema corporal, espacio, tiempo y orientación como saber ineludible de la Educación Física de la educación inicial.

He aquí entonces, que la visión desarrollista de la Educación Física Argentina del Nivel Inicial se encuentra construida por los aportes científicos de índole teóricos de la vertiente psicomotricista, empero sus propuestas pedagógicas prácticas, las hallamos aferradas a la Gimnasia Natural propuesta por Villá de Cardozo (1966). En el correr de las líneas, realizaremos un tratado de las características principales de la propuesta que la autora realiza a la Educación Física de la educación inicial.

Susana Villá de Cardozo, publica su libro La Educación Física en el ciclo preescolar en el año 1966, desarrollando aportes teóricos y prácticos concretos para la Educación Física del nivel inicial. Entre sus objetivos podemos hallar: propender el desarrollo armonioso de sus formas corporales, contrarrestar el 
sedentarismo, prevenir malas posturas y permitir la libre expresión de movimientos.

Villá de Cardozo (1966) expresa, que es fundamental en el nivel preescolar hacer hincapié en la naturalidad del movimiento, retrotrayendo al niño y niña a sus formas más placenteras y simples. Por tales motivos, la autora desde la Gimnasia Natural, ofrece propuestas prácticas para el desarrollo de las habilidades motrices, siendo: trepa, salto, equilibrio, correr, traccionar, entre otras.

En función del análisis e interpretación de los libros y artículos correspondientes a la didáctica de la Educación Física en el nivel inicial (Marrazzo y Marrazzo, 1970; Giraldes y Porstein, 1976; González y Gómez, 1978; Gómez e Incarbone, 2001; Gómez, 2004) damos cuenta que sus alegatos teóricos provienen de corrientes educativas psicomotoras, y sus propuestas pedagógicas se hayan sumergidas en las actividades devenidas de la Gimnasia Natural, disfrazadas como juegos.

Sostenemos ésta afirmación, ya que hemos registrado en dichos documentos, que las tareas motrices propuestas para la Educación Física del Nivel Inicial, son catalogadas como juego, empero dentro de ellas, los objetivos se hayan enmarcados por el/la docente, impidiendo el despliegue del jugar en la niñez. Por tal motivo, encontramos atravesada a la perspectiva recreacionista (Mansi, 2018) de la Educación Física, desde la cual se arraiga al juego como estrategia metodológica para la enseñanza de contenidos curriculares (Rivero, 2011).

Desde la DGCyE (2001) nos aportan que en la década de '80 comienza en el Nivel Inicial una etapa de replanteos y cuestionamientos ideológicos, orientando las prácticas hacia la revalorización de la didáctica del juego. De tal manera, la Educación Física, tratando de acomodarse a los nuevos discursos pedagógicos, sostiene su legitimidad como facilitadora de juegos para el desarrollo y enriquecimiento de la motricidad de las niñas y los niños. Aparece entonces, " $(\ldots)$ una peligrosa tendencia a considerar que ser profesor de Educación Física consiste básicamente en dominar un conjunto de juegos con los que trabajar todos los contenidos de la asignatura" (López Pastor, 2004, p. 5). Siendo así, que la bibliografía correspondiente a la didáctica de la Educación Física en la educación inicial se transforma en un recetario de juegos de resistencia, de cooperación, del esquema corporal, de expresión, de juegos individuales, grupales, y de habilidades motoras básicas.

Concluyendo este tramo del escrito, diremos que la perspectiva desarrollista, atravesada por la visión recreacionista, crea sus escenarios de enseñanza tomando al juego como herramienta que facilita el aprendizaje de contenidos curriculares, específicamente aquellos donde el desarrollo motor se encuentre como núcleo motivador.

Para continuar caracterizando la visión desarrollista, retomaremos, el contexto socio-político contextual, y diremos que el aumento cuantitativo de la bibliografía específica de la Educación Física del nivel inicial, ha comenzado a darse en la década del '70. Tomando a Guitelman (2006) durante el autollamado Proceso de Reorganización Nacional, aparece como política de estado la despolitización de los contenidos curriculares. Dicha idea no se encuentra alejada de la política de turno, sino que (co)rrespone a una ideología en particular. La corriente de la modernización pedagógica dispuesta durante la dictadura iniciada en 1976, ha otorgado un énfasis a los principios de eficiencia en la educación argentina, es decir, que el último golpe de estado cívico-militar ha buscado la 
desacralización de los contenidos y la militarización del sistema educativo, al mismo tiempo que combinó la visión eficientista (Guitelman, 2006).

En base a las manifestaciones de la autora, las propuestas educativas se han encontrado bajo tres valores dominantes: la eficiencia, la eficacia y el rendimiento. La visión desarrollista contiene en su trasfondo dichos valores, bajo sus propuestas de tareas motrices, la enseñanza encontraba su foco en el aprendizaje de técnicas de movimiento por parte de las niñas y niños. Las conductas motoras debían ser observables, objetivables, medibles y evaluables, siguiendo un único modelo posible de moverse esperado por el/la docente.

[...] resurgió un planteo tecnicista que proporcionó un entrenamiento para las tareas... así en la escuela como afuera... en la vida social. Cobró amplia dimensión el aprender a hacer, en forma descontextualizada y parcelada" (Bauso y Merciai, 2017, p. 154).

Si tomamos a González y Gómez (1978), quienes proponen tareas motrices para el desarrollo de habilidades motoras básicas, veremos que sus consignas se encuentran simuladas bajo un discurso propositivo: ¿Quién puede picar la pelota con una mano? ¿Y ahora con las dos? ¿Quién se anima a trepar? ¿Alguien se atreve a saltar los aros? (González y Gómez, 1978). ${ }^{3}$

Si generamos reflexiones sobre el telón de fondo de las consignas, las cuales se disfrazan de progresistas, nos demuestran cómo continúan afianzando las conductas motrices infantiles exitistas hacia la Educación Física infantil. Y por otro lado, damos en evidencia que mediante las consignas sólo era permitido moverse como el/la docente lo solicitase.

El planteo tecnicista se haya en las propuestas devenidas de la perspectiva desarrollista, y si ahondamos aún más, daremos cuenta que el núcleo de los encuentros de Educación Física los hallamos bajo el cumplimiento o no de los objetivos propuestos por el/la docente:

\begin{abstract}
Desde esta mirada, que enfatizaba el orden como objetivo supremo, una concepción pedagógica tecnicista se constituyó en el instrumento más idóneo para generar y/o fortalecer un perfil docente en el que el maestro surgiera como un simple ejecutor de tareas especificadas en los diseños curriculares. (Bauso y Merciai, 2017, p. 154).
\end{abstract}

En consecuencia, la tarea docente se ha encontrado orientada a facilitar un sinfín de propuestas de índole práctica, donde las niñas y los niños debían ser ejecutores de las destrezas motrices (eficientes y eficaces) solicitadas por el/la docente, quien mediante la observación directa ha de pretender cumplimentar los contenidos desarraigados de los diseños curriculares.

\footnotetext{
Esta visión utilitaria clasifica los saberes y las prácticas en general, a partir de valoraciones de utilidad. Es útil lo que pueda ser productivo, eficiente, rentable y toda educación que los enseñe [...] La eficiencia de la enseñanza se mide en función de los cambios "observables" que produce en los alumnos y de la medida en que éstos consiguen las metas propuestas (Rodríguez, 2008, p. 6).
} 
Es en tal caso, que aparece la gobernabilidad de los cuerpos infantiles mediante las prácticas corporales de la educación inicial.

Desde allí, nos atrevemos a decir, que la Educación Física desarrollista del nivel inicial, que hace sus bases sobre el rendimiento motor infantil, se encuentra legitimada bajo la pedagogía de los objetivos la cual subsume el aprendizaje a ciertas conductas observables, que se establecen en términos operativos y de conducta (Rodríguez, 2008), y sus propuestas pedagógicas se encuentran ancladas al orden cronológico - evolutivo de los niños y las niñas.

En función de lo narrado hasta el momento, concluiremos diciendo que la visión desarrollista de la Educación Física del nivel inicial en Argentina, construye sus legitimaciones bajo los aportes cientificista de la corriente educativa psicomotora (Villa, 2006), y las propuestas prácticas de la Gimnasia Natural, devenidas de las ideas de Liselott Diem, siendo sus propósitos: el desarrollo de las habilidades motoras, la resolución motriz de las situaciones problemáticas, el acrecentamiento del acervo motor, la formación física básica, la enseñanza de nuevas conductas motrices, la formación corporal, el perfeccionamiento de las habilidades motoras naturales de la niñez, la combinación de habilidades motoras en simultáneo y la coordinación de las destrezas motrices en el tiempo, el espacio y con los compañeros y compañeras. Desencontrando fundamentaciones teóricas, y propuestas donde los/as niños/as puedan realizar acciones espontáneas con características personales y subjetivas, y desplegar el juego y el jugar como derecho de la infancia.

\section{Conclusiones}

La Educación Física Argentina del nivel inicial, se ha encontrado legitimada bajo distintas corrientes teóricas y prácticas, que le han ofrecido representatividad dentro de los ámbitos escolares y formación docente. Hemos registrado, que la perspectiva desarrollista, ha intentado conformarse como visión autónoma, ofreciendo ser sostenida en el nivel inicial. Empero sus bases (tanto prácticas como teóricas) se han encontrado - y encuentran - bajo los alegatos de la educación psicomotora, en especial de corte de Le Boulch (1978) y de la Gimnasia Natural (Villá de Cardozo, 1966).

Sus propósitos han de ser meramente funcionales al desarrollo motor infantil, ofreciéndole a la Educación Física de la educación inicial la función de facilitar el desarrollo de las habilidades motoras básicas en la niñez, construyendo de tal manera una infancia productora de destrezas motrices, generando un gobierno de los cuerpos infantiles.

Diremos para concluir, que la perspectiva desarrollista, registrada en la bibliografía competente a la Educación Física del nivel inicial a partir de la década de 1970 hasta la actualidad, impide el despliegue del juego y jugar en la infancia, y el desarrollo de la disponibilidad corporal, por evidenciar un fuerte afianzamiento al tecnicismo pedagógico, desde las cuales la eficiencia, la eficacia y el rendimiento infantil se muestran como valores dominantes. 


\section{Bibliografía}

Aisenstein, A. (2006). La educación física escolar en Argentina. Conformación y permanencia de una matris disciplinar, 1880 - 1960. (Tesis inédita de doctorado). Buenos Aires: Universidad de San Andrés.

Aisenstein, A., y Scharagrodsky, P. (2006). Tras las huellas de la Educación Física Escolar Argentina. Cuerpo, género y pedagogía. 1880 - 1950. Buenos Aire s: Prometeo.

Bauso, A. y Merciai, A. (2017). (Etapa 1974-1983) El Proyecto Educativo Autoritario en A. Simon, R. Ponce y A. Encabo (comp) Apuntes de Historia y Política del Nivel Inicial (pp. 133-170). Buenos Aires: EdUNLu.

Beer, D. (2013). La cultura del Instituto Nacional de Educación Física de Buenos Aires durante la última dictadura militar; deportivización, autoritarismo y resistencia. En Actas del $10^{\circ}$ Congreso Argentino y $5^{\circ}$ Latinoamericano de Educación Física y Ciencias.

Bracht, V. (1996) Educación Física y Aprendizaje Social. Córdoba: Vélez Sarsfield.

Denzin, N. y Lincoln, Y. (2011). Manual de investigación cualitativa. Volúmen I. Barcelona: Gedisa.

Dirección General de Cultura y Educación (2001) Antecedentes históricos de la Educación Inicial - República Argentina. Recuperado en www.servicios.abc.gov.ar/lainstitucion/sistemaeducativo/educacióninicial/

Falkenbach, A., Drexsler, G., Werle, V. (2006). Investigando a acao Pedagógica de Educacao Física na Educacao Infantil. Movimento, 12(1), 81-103.

Fernández Pais, M. (2019) Historia y Pedagogía de la Educación Inicial en la Argentina. Desde el proyecto sarmientino hasta los inicios del siglo XIX. Buenos Aires: HomoSapiens.

Galak, E., y Gambarotta, E. (2015). Cuerpo, educación, política. Tensiones epistémicas, históricas y prácticas. Ciudad Autónoma de Buenos Aires: Biblos.

Giraldes, M. y Porstein, A. (1976). El olvidado nivel preescolar. Revista Stadium, 60(10), 45-48.

Gómez, J., e Incarbone, O. (2001). Juego y Motricidad. Buenos Aires: Noveduc.

Gómez, R. (2004). La enseñanza de la Educación Física en el nivel inicial y primer ciclo de la eb. Una didáctica de la disponibilidad corporal. Buenos Aires: Stadium.

González, L., y Gómez, J. (1978). La Educación Física en la Primera Infancia. Buenos Aires: Stadium.

Guitelman, P. (2006). La infancia en dictadura. Modernidad y conservadurismo en el mundo de Billiken. Buenos Aires: Prometeo.

Le Boulch, J. (1978). Hacia una ciencia del movimiento humano. Introducción a la psikokinética. Buenos Aires: PAIDÓS.

López Pastor, V.M. (2004). Una Mirada Crítica sobre las prácticas en Educación Física. Novedades Educativas, 157, 4-5

Mansi, D. (2018). Construcción Histórica de la Educación Física Argentina del Nivel Inicial. Tesis Inédita de Maestría. Universidad de Flores, Buenos Aires. 
Marrazo, T. y B. de Marrazo, M. (1970). Guía de educación física para los jardines de infantes. Batería de planes para los niveles de cuatro y cinco años. Buenos Aires: Ciordia.

Orbuch, I. (2016). Peronismo y Educación Física. Políticas públicas entre 1946 y 1955. Buenos Aires: Miño y Dávila.

Puiggrós, A. (1992). Historia de la educación Argentina III. Escuela, Democracia y Orden (1916 - 1943). Buenos Aires: Galerna.

Rivero, I. (2011). El juego en las planificaciones de Educación Física. Intencionalidad educativa y prácticas docentes. Buenos Aires: Noveduc.

Rodríguez, Norma (2008). Saberes y prácticas corporales en la escuela. Los contenidos como propuesta. $V$ Jornadas de Sociología de la UNLP. Universidad Nacional de La Plata. Facultad de Humanidades y Ciencias de la Educación. Departamento de Sociología, La Plata.

Rozengardt, R. y Acosta, F. (2006). Historia de la Educación Física y sus Instituciones: continuidades y rupuras. Buenos Aires: Miño y Dávila.

Samaja, J. (1999). Epistemología y Metodología. Elementos para una teoría de la investigación científica. Buenos Aires: Eudeba.

Scharagrodsky, P. (2004). El padre de la Educación Física Argentina: fabricando una política corporal generizada (1900-1940). Perspectiva 22, № especial (83119).

Scharagrodsky, P. (2008). Gobernar es ejercitar. Fragmentos históricos de la Educación Física en lberoamérica. Buenos Aires: Prometeo.

Scharagrodsky, P. (2011). La invención del "homo gymnasticus". Fragmentos históricos sobre la educación de los cuerpos en movimiento en occidente. Buenos Aires: Prometeo.

Scharagrodsky, P. (2014). Miradas médicas sobre la cultura física en Argentina 1880 - 1970. Buenos Aires: Prometeo.

Scharagrodsky, P. (2015). El Sistema Argentino de Educación Física. Entre el cientificismo, la higienización, el eclecticismo y la argentinidad. Revista Brasileira de Ciências do Esporte, 37 (2). 158-164.

Valles, M. (1999). Técnicas Cualitativas de Investigación Social. Reflexión metodológica y práctica profesional. España: Editorial Síntesis.

Villá de Cardozo, S. (1966). La educación física en el ciclo preescolar. Buenos Aires: Nubef.

Villa, M. (2006). La educación física en la infancia. Historia crítica de la especialidad "educación física infantil" en el Profesorado de Educación Física de la Universidad Nacional de La Plata (1962 - 2000). (Tesis inédita de Maestría). Santiago: Universidad Academia de Humanismo Cristiano.

Ynoub, R. (2015). Cuestión de método. Aportes para una metodología crítica. Tomo I. México D.F: CENGAGE Learning. 


\section{Notas}

${ }^{1}$ La misma es consecuente a los resultados de la tesis de Maestría titulada Construcción Histórica
de la Educación Física Argentina en el Nivel Inicial (Mansi, 2018).
${ }^{2}$ El documento difundido se titulaba "Subversión en el ámbito educativo (conozcamos a nuestro
enemigo)" (MCyE, 1977). Con un total de 77 páginas, se desarrollan 4 capítulos: conceptos
generales de la subversión, organizaciones subversivas que operan en el ámbito educativo,
estrategia particular de la subversión en el ámbito educativo y como último capítulo, construir el
futuro.
${ }^{3}$ Dicho libro ha sido de gran significatividad para la Educación Física del Nivel Inicial. Se registra
una venta de 30 mil ejemplares hasta el año 2010 (según editorial Stadium), y colocado como
bibliografía obligatoria en las cátedras de Educación Física Infantil de distintas instituciones de
formación docente en Argentina. 